\title{
BISTATIC FREQUENCY-SWEPT MICROWAVE IMAGING MEASUREMENT SYSTEM
}

\author{
Ding-Bing Lin and Tah-Hsiung $\mathrm{Chu}^{*}$ \\ Electrical Engineering Department, National Taiwan University \\ Taipei, Tawan, Republic of China
}

\section{Introduction}

It has been of long standing interest in inverse scattering problems to develop analytical method and measurement system that enable one to use the information in the measured electromagnetic field to infer the geometrical shape, or the material characteristics of the unknown scattering object [1]. In this paper, experimental studies of the frequency-swept microwave image reconstruction scheme for perfectly conducting objects in a bistatic backward scattering arrangement are presented.

\section{Measurement System and Calibration Procedure}

Shown in Fig. 1 is the developed experimental system to measure the wideband bistatic scattering data of test object in a backward scattering arrangement. In the measurement system, an Arra X820 rectangular horn antenna is used for transmitting wide-band signals, and an open-ended WR-90 rectangular waveguide mounted on a linear scanner is used as the receiving probe. The linear scanner consists of a stepping motor for moving the receiving probe along the dashed line shown in Fig. 1 to synthesize a linear receiving array. The stepping motor driver is controlled by HP3852A data control unit. The measurement system also includes Hughes $8010 \mathrm{H}$ traveling wave tube amplifier (TWTA), Avantek AWT-18235 low noise amplifier (LNA), and HP8510B network analyzer. The measurement system is automated with MicroVax 3500 via an IEEE-488 interface bus to perform instrument control, data acquisition, and signal processing.

The calibration procedures of the developed measurement system to be described in the following is based on the plane wave expansion [2]. By considering the error associated with both the receiving probe response and the measurement system frequency response as a filter designated as $\tilde{R}\left(k_{x}, k_{0}\right)$ in the plane wave spectrum (PWS) domain, the measured wide-band scattered field over the linear array located at $z=-d$ can be expressed as [3]

$$
U_{m}^{\prime}\left(x, z=-d, k_{0}\right)=\frac{1}{2 \pi} \int \tilde{A}_{o}\left(k_{x}, k_{0}\right) e^{\left.-j \mid k_{x} x+(-\gamma)(-d)\right]} d k_{x}
$$

where

$$
\tilde{A}_{o}\left(k_{x}, k_{0}\right)=\tilde{I}\left(k_{x}, k_{0}\right)+\frac{-j \mathcal{K}}{2 \gamma} \bar{O}\left(k_{x},-\gamma-k_{\mathrm{c}}\right) \tilde{R}\left(k_{x}, k_{0}\right)
$$

is defined as the PWS of the wave represented by $(1), \ddot{J}\left(k_{x}, k_{0}\right)$ is the PWS of the additive isolation error resulting from the residual reflection of the empty 


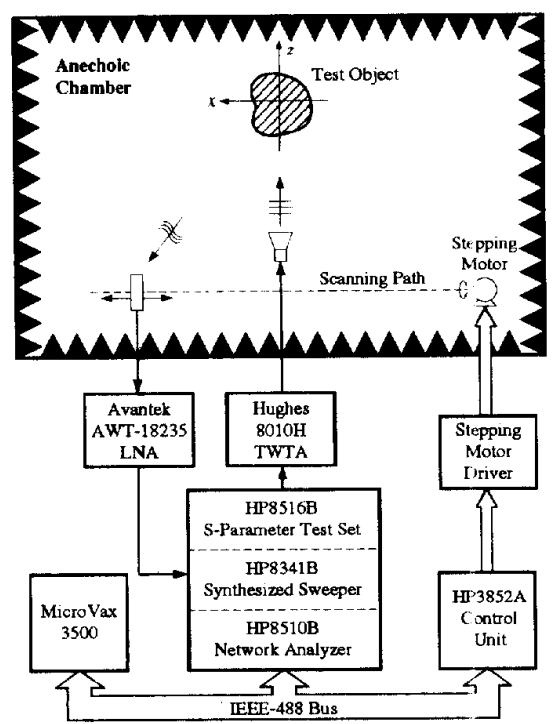

Fig. 1: Automated wide-band bistatic scattering measurement system.

room and the coupling between transmitting and receiving antennas, $\mathcal{K}$ is the scaling factor, $\tilde{O}\left(k_{x},-\gamma-k_{0}\right)$ is the object Fourier space data, and the arguments of $\tilde{O}(\mathbf{k})$ are related by

$$
k_{x}^{2}+\left(k_{z}+k_{0}\right)^{2}=k_{0}^{2} .
$$

By one-dimensional Fourier transforming the measured scattered field represented by (1) in $x$-direction, one can obtain

$$
\tilde{U}_{m}^{*}\left(k_{x}, z=-d, k_{0}\right)=\tilde{I}\left(k_{x}, k_{0}\right) e^{-j \gamma d}+\frac{-j \mathcal{K}}{2 \gamma} e^{-j \gamma d} \tilde{O}\left(k_{x}-\gamma-k_{0}\right) \tilde{R}\left(k_{x}, k_{0}\right) \text {. (4) }
$$

Therefore, from (4) the calibrated Fourier space data can be calculated as

$$
\tilde{O}\left(k_{x},-\gamma-k_{0}\right)=\frac{\tilde{U}_{m}^{e}\left(k_{x}, z=-d, k_{0}\right)-\tilde{I}\left(k_{x}, k_{0}\right) e^{-j \gamma d}}{\tilde{U}_{\text {ref }}^{e}\left(k_{x}, z=-d, k_{0}\right)-\tilde{I}\left(k_{x}, k_{0}\right) e^{-j \gamma d}} \tilde{O}_{r e f}\left(k_{x},-\gamma-k_{0}\right)
$$

where $\tilde{U}_{\text {ref }}^{:}\left(k_{x}, z=-d, k_{0}\right)$ and $\tilde{O}_{r e f}\left(k_{x},-\gamma-k_{0}\right)$ are the measured and calculated Fourier space data of a reference object used to eliminate the error terms given in (4).

III. Frequency Diversity Technique

According to (3), the calibrated Fourier space data shown by (5) yields a semicircular slice centered at $\left(0,-k_{0}\right)$ with radius $k_{0}$ in the two-dimensional 


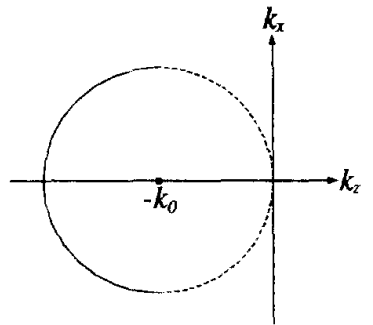

Fig. 2: Fourier slice acquired in the backward scattering arrangement with single-frequency (wavenumber $k_{0}$ ) plane wave illumination.

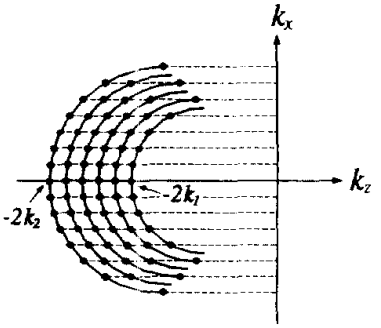

Fig. 3: Fourier space data acquired in the backward scattering arrangement using frequency diversity technique with $k_{0}$ stepped from $k_{1}$ to $k_{2}$

Fourier space $\widetilde{O}(\mathbf{k})$ of the scattering function as the semicircle denoted by the solid line shown in Fig. 2. In order to reconstruct the scattering object image with high resolution, a large portion of Fourier space data can be acquired by linearly stepping the frequency of incident plane wave from $f_{1}$ to $f_{2}$. Both the radius and the center of semicircular slice shown by (3) will vary from $k_{1}$ and $\left(0,-k_{1}\right)$ to $k_{2}$ and $\left(0,-k_{2}\right)$ accordingly. The semicircle denoted by the solid line in Fig. 2 then extends to a fan-shaped section in the Fourier space as shown in Fig. 3. Therefore, the two-dimensional Fourier inversion of band-pass version of the limited Fourier space data yields the image of test scattering object. This is known as the frequency diversity technique [3].

\section{Experimental Results}

Based on the above calibration procedures and frequency diversity technique, given in this section are the experimental results of two different types of scattering object: (a) discrete line scatterers of four distributed metallic thin cylinders with length $113 \mathrm{~cm}$ and radius $a=0.5 \mathrm{~cm}$, and (b) a complex scattering object of 1:100 metal covered B-52 scale model aircraft, for demonstrating the performance of the developed frequency-swept bistatic microwave imaging system. In the measurement, a $153.6-\mathrm{cm}$-long linear scanning positioner located at about $z=-95 \mathrm{~cm}$ with reference to the center of test object positioner. The linear receiving array is synthesized by moving an open-ended WR-90 rectangular waveguide at 128 equally spaced position. The frequency is stepped from 7.5 to $12.5 \mathrm{GHz}$ for 51 frequency points. The reference object used for calibration is a metallic cylinder with length $113 \mathrm{~cm}$ and radius $a=0.5 \mathrm{~cm}$.

In the type (a) test object, the range of $k a$ is $0.79-1.31$ radians, hence the thin cylinders can be treated as four line scatterers. Results of the Fourier space data and reconstructed image are shown in Fig. 4(a) and (b). The reconstructed image is shown in good agreement with the distribution of four 


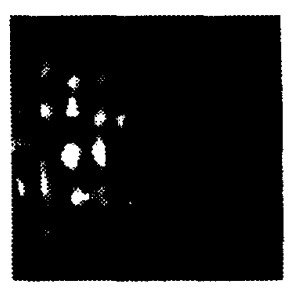

(a)

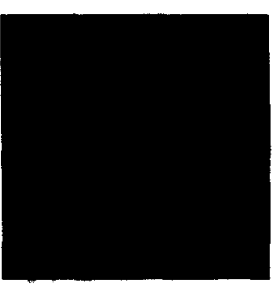

(b)

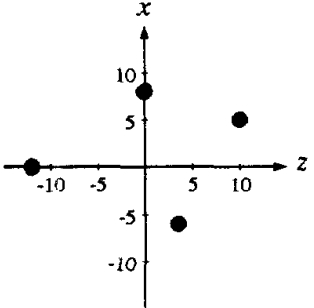

(c)

Fig. 4: (a) Fourier space data and (b) reconstructed image of four thin cylinders with (c) geometries at $(-12,0) \mathrm{cm},(0,8) \mathrm{cm},(3.5,-6) \mathrm{cm}$, and $(10,5) \mathrm{cm}$ respectively.

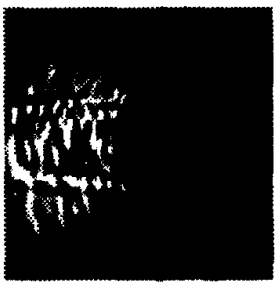

(a)

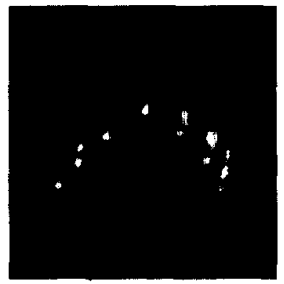

(b)

Fig. 5: (a) Fourier space data and (b) reconstructed image of a 1:100 B-52 scale model aircraft.

thin cylinders given in Fig. 4(c). For the complex object of a B-52 scale model aircraft, it consists of discrete and continuous scattering centers contributed to the measured bistatic frequency-swept responses. Results of the Fourier space data and reconstructed image are shown in Fig. 5(a) and (b). The reconstructed image clearly indicates those scattering centers on the illuminated surface of test aircraft observed by the linear array.

\section{References}

[1] H. P. Baltes, Ed., Inverse Scattering Problems in Optics. New York: Springer-Verlag, 1980.

[2] J. Appel-Hansen, "Antenna measurements," in The Handbook of Antenna Design, vol. 1. London: Peregrinus, ch. 8, 1982.

[3] D. B. Lin and T. H. Chu, "Bistatic frequency-swept microwave imaging: principle, methodology and experimental results," to be published in IEEE Trans. Microwave Theory Tech.. 